\title{
Sources of variation in diets of harp and hooded seals estimated from quantitative fatty acid signature analysis (QFASA)
}

\author{
Strahan Tucker ${ }^{1,5, *}$, W. Don Bowen ${ }^{2}$, Sara J. Iverson ${ }^{1}$, Wade Blanchard ${ }^{3}$, \\ Garry B. Stenson ${ }^{4}$ \\ ${ }^{1}$ Department of Biology, Dalhousie University, 355 Oxford Street, Halifax, Nova Scotia B3H 4J1, Canada \\ ${ }^{2}$ Population Ecology Division, Bedford Institute of Oceanography, Department of Fisheries and Oceans, 1 Challenger Drive, \\ Dartmouth, Nova Scotia B2Y 4A2, Canada \\ ${ }^{3}$ Department of Mathematics and Statistics, Dalhousie University, 355 Oxford Street, Halifax, Nova Scotia B3H 4J1, Canada \\ ${ }^{4}$ Northwest Atlantic Fisheries Centre, Department of Fisheries and Oceans, PO Box 5667, St John's, Newfoundland A1C 5X1, \\ Canada
}

${ }^{5}$ Present address: Pacific Biological Station, Department of Fisheries and Oceans, 3190 Hammond Bay Road, Nanaimo, British Columbia V9T 6N7, Canada

\begin{abstract}
Inter-specific competition for prey is thought to influence the structure of ecological communities and species niche breadth. Harp seals Pagophilus groenlandicus and hooded seals Cystophora cristata are geographically overlapping and highly migratory predators in the North Atlantic ocean. Hooded seals are known to dive deeper and longer than harp seals and are more closely associated with the continental shelf edge and deep ocean. Quantitative fatty acid (FA) signature analysis (QFASA) was recently developed to estimate the species composition of diets by statistically comparing FA signatures of predator adipose tissue with that of potential prey. Using QFASA,

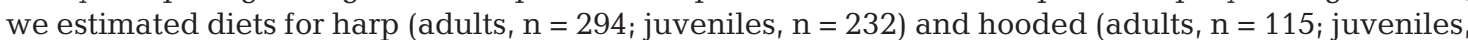
$\mathrm{n}=38$ ) seals from the pre- and post-breeding periods between 1994 and 2004. We found evidence of inter- and intra-specific variation in diets, diet quality and breadth, reflecting different foraging tactics. Harp seal diets were comprised predominantly of amphipods, Arctic cod, capelin, herring, sand lance and redfish. Hooded seal diets were composed primarily of amphipods, Atlantic argentine, capelin, euphausiids and redfish. Relative to the other species, harp seals consumed twice the proportion of amphipods, while hooded seals consumed 3 times the proportion of redfish; percentages of capelin were similar. QFASA provided new evidence of the importance of amphipods in the diets of both species and of the pronounced differences in the proportions of pelagic forage fish between demographic groups.
\end{abstract}

KEY WORDS: Harp seal - Pagophilus groenlandicus Hooded seal · Cystophora cristata - Diet segregation · Quantitative fatty acid signature analysis · QFASA

\section{INTRODUCTION}

Harp seals Pagophilus groenlandicus and hooded seals Cystophora cristata are abundant, wide-ranging piscivores found throughout the North Atlantic Ocean (Stenson et al. 1997, 2002) where they are sympatric over much of their range. Although both species have been implicated in the declines of commercial fish stocks or in their failure to recover (see Sinclair \& Murawski 1997), estimating the impact of these species on fish-stock dynamics requires integrating predation pressure spatially and temporally (e.g. Hammill \& 
Stenson 2000). Such models require information on predator abundance, spatial and temporal distribution, population structure, consumption rates and diet composition. However, we have a relatively poor understanding of factors influencing diet throughout the entire range of these species. Studies on other pinniped species have shown that different age and sex classes can differ in their spatial and temporal patterns of feeding (e.g. Beck et al. 2003, Field et al. 2005, Breed et al. 2006). Moreover, through the application of novel biochemical tracer techniques using fatty acids (FA) and stable isotopes, there is emerging evidence for large intra-specific variation in diet amongst pinniped species related to ontogeny and sex (e.g. Beck et al. 2007, Tucker et al. 2007). Indeed, in a previous study (Tucker et al. 2009) we found significant differences in FA composition of blubber both between and within harp and hooded seals, although specific sources of diet variation were not identified.

Inter-specific competition for prey is thought to influence the structure of ecological communities and species niche breadth (Polis 1984). Within species, various explanations have been proposed to account for diet divergences, such as differential energetic requirements, sex-specific costs of reproduction, ontogenetic niche shift, resource polymorphism or minimising inter-specific competition (reviewed in Bolnick et al. 2003). Differential resource use by the sexes, observed in both size dimorphic (e.g. Beck et al. 2007) and monomorphic (e.g. Lewis et al. 2002) species, has been linked to sex-specific reproductive costs. Competition between the sexes also may be minimised by the selection of different prey or by spatial/temporal segregation in feeding between males and females (e.g. Breed et al. 2006, Beck et al. 2007).

Apart from temporal or spatial segregation in resource use, these hypotheses do not make specific predictions about diet selection or the subsequent properties associated with that diet (i.e. the energetic quality and costs related to capture and digestion). Harp and hooded seals offer an opportunity to explore, in a comparative manner, intrinsic and extrinsic factors influencing overall breadth and quality of diet in marine carnivores beyond their perceived importance with respect to impact on commercial fish stocks. There are 2 reasons for this. First, harp and hooded seals exhibit differences in life history, foraging behaviour and body size. Second, North Atlantic ecosystems have undergone profound changes in species' abundance and distribution due to overfishing and environmental variability, such as trends in ocean temperature (Carscadden et al. 2001, Rose 2004). This contrast in the environment allows us to evaluate interactions with intrinsic factors influencing diet.
Harp seals are only slightly size-dimorphic with an average adult mass of $130 \mathrm{~kg}$ and males being 5 to $10 \%$ larger than females (Hammill et al. 1995, Hammill \& Stenson 2000). Hooded seals are more size-dimorphic, with adult males being approximately 1.5 times larger than females and 2.3 times larger than adult harps (Chabot et al. 2006). Although both species are wide-ranging and exhibit long-distance seasonal migrations, harp seals mainly inhabit the continental shelf (Stenson \& Sjare 1997, Folkow et al. 2004), while hooded seals are more strongly associated with the continental shelf edge and the deep ocean (Folkow \& Blix 1999). Harp and hooded seals also differ in their diving behaviour. Most harp seal dives are $<50 \mathrm{~m}$, although dives to $200 \mathrm{~m}$ have been recorded (Stenson \& Sjare 1997, Folkow et al. 2004). By contrast, hooded seals regularly dive $>100 \mathrm{~m}$, with dives often exceeding 1000 m (Folkow \& Blix 1999). Data from stomachcontent analysis suggest that harp seals consume a mixed diet of pelagic forage fish and invertebrates such as capelin Mallotus villosus, Arctic cod Boregadus saida, herring Clupea harengus, euphausiids and amphipods (Lawson et al. 1995, Lawson \& Stenson 1997). Limited data on hooded seal diets suggest they feed on a mixture of deeper-water pelagic and demersal species such as halibut Hippoglossus hippoglossus, redfish (Sebastes sp.) and squid, with smaller quantities of herring, capelin, Atlantic cod Gadus morhua and Arctic cod (Kapel 1995, Hammill \& Stenson 2000, Potelov et al. 2000, Haug et al. 2004, 2007). However, the majority of samples for both species have been collected during winter, either before or after the breeding season, and mostly from relatively nearshore locations, potentially biasing our impression of diets.

Quantitative FA signature analysis (QFASA) provides estimates of the species composition of diets (Iverson et al. 2004, 2007, Beck et al. 2007), as deposited FAs represent the assimilated portion of diet over several weeks or months (Iverson et al. 2004). Dietary FAs are deposited in animal adipose tissue in a predictable manner; there are limits on polyunsaturated FA biosynthesis in higherorder consumers (Iverson 1993); and prey species differ in their FA signatures. Therefore, proportional estimates of diet composition can be made by statistically estimating the mixture of prey species that best matches a predator's FA signature, after accounting for predator metabolism effects (Iverson et al. 2004). This model has been validated in captive studies of seals, mink and seabirds (Iverson et al. 2004, 2007, Nordstrom et al. 2008) and independently corroborated for free-ranging grey seals Halichoerus grypus (Tucker et al. 2008) and seabirds (Iverson et al. 2007) through stable isotope analysis and stomach-content analysis, respectively.

We used QFASA to study demographic, temporal and spatial sources of variation in diets of harp and 
hooded seals. Diet estimates were subsequently evaluated with respect to quality in terms of energy density and niche breadth. Our objective was to test for segregation in diets among harp and hooded seals as predicted by species differences in diving behaviour. Given sex-specific costs of reproduction, we also expected the species composition and quality of adult diets to differ between males and females of both species. Given their greater body-size dimorphism, we expected hooded seals to exhibit a greater sex-specific divergence in diets. We expected larger, older animals to consume a lower-quality, yet more diverse, diet because of larger gut capacity and the potential for higher throughput than smaller seals. Finally, as both species are seasonally migratory and therefore likely encounter different prey assemblages, we expected seasonal and geographic effects on diets.

\section{MATERIALS AND METHODS}

Sampling of seals. Blubber samples were obtained from harp and hooded seals killed under permit along the northeast coast of Newfoundland and southern Labrador between November and May from 1994 to
Table 1. Pagophilus groenlandicus (Erxleben, 1777) and Cystophora cristata (Erxleben, 1777). Sample sizes (na = not available) from pre-and postbreeding periods and different locations. Blubber biopsy samples from livecaptured animals in the pre-breeding period were taken from 'the Front' (see 'Sampling of seals'), while biopsy samples in the post-breeding period were taken from East Greenland

\begin{tabular}{|c|c|c|c|c|c|c|}
\hline \multirow{3}{*}{ Age class } & \multicolumn{6}{|c|}{ - Sample sizes } \\
\hline & \multicolumn{3}{|c|}{ Pre-breeding } & \multicolumn{3}{|c|}{ — Post-breeding } \\
\hline & Inshore & Offshore & Biopsy & Inshore & Offshore & Biopsy \\
\hline \multicolumn{7}{|l|}{ Harp } \\
\hline Juvenile & 144 & 41 & na & 11 & 36 & na \\
\hline Adult female & 97 & 25 & 31 & 11 & 25 & na \\
\hline Adult male & 47 & 39 & na & 8 & 11 & na \\
\hline \multicolumn{7}{|l|}{ Hooded } \\
\hline Juvenile & 31 & 2 & na & na & 2 & 3 \\
\hline Adult female & 17 & 11 & 8 & 3 & 7 & 9 \\
\hline Adult male & 27 & 13 & 2 & 3 & 10 & 5 \\
\hline
\end{tabular}

2004 by licensed and experienced seal hunters or scientific personnel from the Department of Fisheries and Oceans Canada (Sjare et al. 2004) (Fig. 1, Tables 1 \& 2). Evidence from grey seals (Cooper 2004) suggests that blubber lipid composition in phocid seals is uniform throughout the trunk of the body. Nevertheless, for consistency we took samples from the same location in all animals. A full-depth blubber sample $(\sim 0.5 \mathrm{~kg}$ from skin to underlying muscle) was taken from the posterior mid-flank of each seal, placed in a Whirlpak ${ }^{\circledR}$ and frozen. The age of seals was determined to the nearest

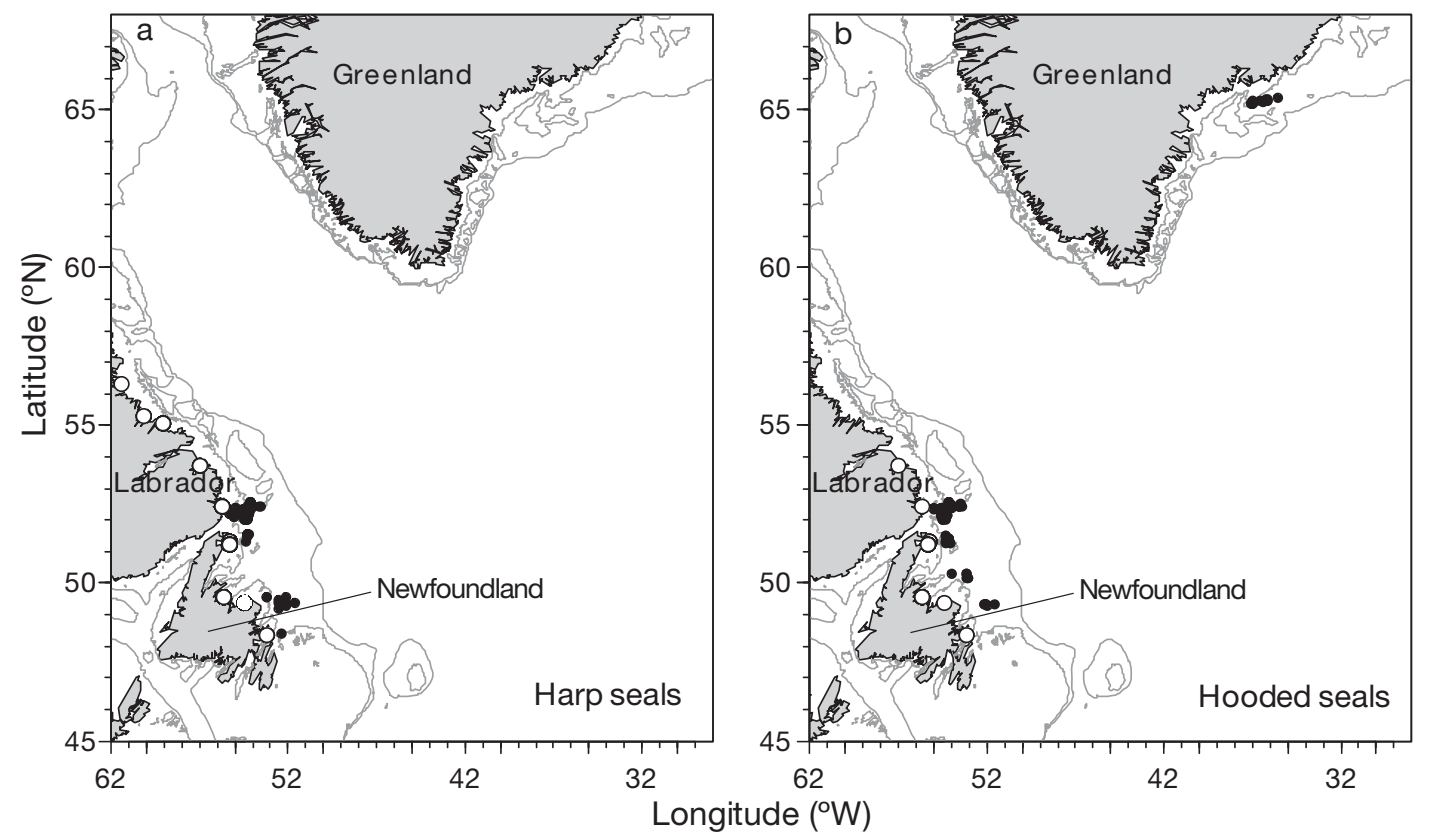

Fig. 1. Pagophilus groenlandicus and Cystophora cristata. Sampling locations for (a) harp ( $\mathrm{n}=526)$ and (b) hooded (n = 153) seals (1994 to 2005). O, inshore and $\bullet$, offshore sampling locations. Lines represent the 200,500 and $2500 \mathrm{~m}$ isobaths 
Table 2. Pagophilus groenlandicus (Erxleben, 1777). Sample sizes from individual years (1994 to 2004). Samples are pooled across seasons and sampling locations

\begin{tabular}{|lrrrrrrrr|}
\hline Age class & \multicolumn{7}{c|}{ Year } \\
& 1994 & 1995 & 1996 & 1998 & 2000 & 2002 & 2004 \\
\hline Juvenile & 20 & 30 & 48 & 20 & 16 & 33 & 65 \\
Adult female & 11 & 12 & 23 & 24 & 11 & 40 & 37 \\
Adult male & 23 & 6 & 12 & 15 & 5 & 14 & 30 \\
\hline
\end{tabular}

year by sectioning a lower canine tooth and then counting dentine annuli (Bowen et al. 1983). In addition to the samples from killed seals, full-depth biopsy samples were taken from the flanks of seals live-captured during research at the whelping patch on the pack ice off southern Labrador ('the Front') in March 2004 (31 harp and 12 hooded) and at the moulting area off eastern Greenland during June 2005 (17 hooded). All procedures used in the present study were in accordance with the principles and guidelines of the Canadian Council on Animal Care adopted by the Department of Fisheries and Oceans Canada. In the laboratory, a $0.5 \mathrm{~g}$ core of blubber representing the entire depth of each field-collected sample (i.e. from skin to underlying muscle) was taken. Lipids were quantitatively extracted from all blubber samples using a modified Folch method (Folch et al. 1957, Iverson et al. 2001).

Samples from harp seal juveniles (1 to 4 yr old) and adults ( $\geq 5$ yr old) (Sjare et al. 2004) were grouped by season: pre-breeding (November to March) and postbreeding (April and May). No harp seals were sampled during the summer. Samples were also grouped by sampling location: inshore (defined as $<30 \mathrm{~km}$ from shore), and offshore (defined as $>30 \mathrm{~km}$ from shore; Lawson et al. 1995). Offshore areas are typically over water depths exceeding $200 \mathrm{~m}$ (see Fig. 1). Samples from hooded seal juveniles ( 1 to $5 \mathrm{yr}$ old) and adults ( $\geq 6 \mathrm{yr}$ old) were also grouped into pre- and postbreeding periods (where breeding occurred in midMarch) and sampling area: inshore and offshore as defined above, as well as Greenland.

Sampling of prey. Fishes and invertebrates were collected and frozen during stratified, random, bottomtrawl surveys conducted in the summer in the northwest Atlantic (Northwest Atlantic Fisheries Organization [NAFO] sub-areas $2 \mathrm{~J}, 3 \mathrm{~K}, 3 \mathrm{~L}, 4 \mathrm{~T}$ and $4 \mathrm{~V}$ ) between 1993 and 2002 (Budge et al. 2002). Supplementary samples were collected from deep-water trawl surveys of the Davis Strait (NAFO sub-areas 0A and 1A) in the fall of 2004. Specimens were thawed and fork length or carapace width was measured to the nearest $0.1 \mathrm{~cm}$ and body mass to the nearest $0.1 \mathrm{~g}$. Individual prey were then homogenised and lipids were quantitatively recovered in duplicate from a $\sim 1.5 \mathrm{~g}$ subsample of the homogenate as above.

FA analysis. FA methyl esters (FAME) were prepared using an acidic catalyst (the Hilditch method; see Iverson 1993). Duplicate identification of FAME was performed using temperature-programmed gasliquid chromatography (GLC) (Iverson et al. 1997, 2004). FAs were described by the standard nomenclature of carbon chain length: number of double bonds and location $(\mathrm{n}-\mathrm{x})$ of the double bond nearest the terminal methyl group. Individual FAs were expressed as a mass percent of total FAs. Although 67 FAs are routinely identified, we used 39 FAs of 'dietary' or 'primarily dietary' origin in our analysis (Iverson et al. 2004, Beck et al. 2005), which accounted for $~ 93 \%$ of total FAs by mass (Tucker et al. 2009). Dietary FA are unmodified FA that are directly deposited in adipose tissue; primarily dietary FA are modified at some point between absorption and deposition but their levels in a predator are highly influenced by consumption of specific prey.

Diet estimation. The diet of individual seals was estimated using QFASA (Iverson et al. 2004). A description of data inputs used in the estimation procedure is given in Tucker (2007) and in the supplement in MEPS Supplementary Material at www.int-res.com/ articles/suppl/m384p287_app.pdf. Briefly, the statistical procedure estimates the mixture of prey FA signatures that minimised the Kullback-Leibler distance between the prey FA mixture and the adjusted FA composition of each seal. Seal FA composition was adjusted for predator FA metabolism using calibration coefficients ( $\mathrm{CC}_{\text {; }}$ see next paragraph). Next, the estimated mixture of prey was converted to an estimate of diet by weighting each prey species by its mean fat content. Standard errors of the estimated diet included sources of variability within (i.e. within prey-species variability in FA composition and fat content) and between seals (Beck et al. 2007). We used a prey library comprised of 2039 individuals representing 24 species to estimate the diets of harp seals and 2289 individuals representing 29 species to estimate the diets of hooded seals.

$\mathrm{CCs}$ account for the effects of predator metabolism on the deposition of FAs (Iverson et al. 2004). We averaged diet estimates of harp seals from 3 model runs, each using a different $\mathrm{CC}$ set. The first run used only the harp seal CCs, the second used the average of grey and harp CCs, and the final used the average of the grey, harp and pup CCs. For each set of CC, the bootstrapping procedure (Beck et al. 2007) was conducted and the 3 estimates of diet were averaged. Similarly for hooded seals, we averaged estimates across 2 model iterations that used the grey-harp CCs and the greyharp-pup CCs. We used an averaging procedure in modelling iterations to incorporate uncertainty in CCs. 
Diet diversity or niche breadth was calculated for each seal's diet using the standardised ShannonWiener index ( $H^{\prime} ;$ Krebs 1999):

$$
H^{\prime}=\left(-\sum_{S}^{j} p_{j} \ln p_{j}\right) / \ln S
$$

where $p_{j}$ is the proportion of prey species $j$ in the diet, and $S$ is the total number of prey consumed across all individuals ( $S=24$ for harps; $S=26$ for hoods). Energy density $\left(E_{\mathrm{d}} ; \mathrm{kJ} \mathrm{g}^{-1}\right)$ of diets was calculated using the lipid composition of prey species.

Dietary overlap was calculated between each ageclass group and between species using the MorisitaHorn index $\left(C_{\mathrm{H}}\right.$ Krebs 1999):

$$
C_{\mathrm{H}}=\left[2\left(\sum_{j}^{n} p_{j k} p_{j 1}\right)\right] /\left[\left(\sum_{j}^{n} p^{2}{ }_{j k}\right)+\left(\sum_{j}^{n} p^{2}{ }_{j 1}\right)\right]
$$

where $p_{j k}$ is the mean proportion of prey type $j$ in the diet of group $k, p_{j 1}$ is the mean proportion of prey type $j$ in the diet of group $l$, and $n$ is the total number of prey consumed by both groups. Degree of dietary overlap is small when $C_{\mathrm{H}}$ is between 0 and 0.29 , medium when $C_{\mathrm{H}}$ is between 0.30 and 0.59 and large when $C_{\mathrm{H}}>0.60$.

Statistical analysis. To test for the effects of seal species, sex of seals, age class, season, sampling area and year (in the case of harp seals) on the diets of seals, we used a randomisation procedure (Efron \& Tibshirani 1998). Two-way multivariate analyses of variance (MANOVAs; R v. 2.3.1; R Development Core Team) were performed to generate test statistics for main effects and interactions in pairwise comparisons. We then randomly permuted the factor labels 10000 times to build a permutation distribution rather than compare test statistics to normal distributions. Significance levels were then computed by determining the number of times the reference distribution gave a test statistic equal to or greater than the observed value. If the overall test was significant, we proceeded with pairwise post hoc tests. Post hoc univariate and multivariate $t$-tests were also compared to the permutation distributions to determine where the significant differences occurred. We also used MANOVA to test for main effects of sex, age class, season, area and species on $E_{\mathrm{d}}$ and $H^{\prime}$ (SPSS v. 11.5). For the sake of brevity, we report only significant interaction terms.

\section{RESULTS}

\section{Inter-specific differences}

There were significant differences in estimated diets between species, age class, sex, area and season (all $\mathrm{p}=$ 0.0001) (Tables 3 \& 4, Fig. 2). We identified 24 prey species in harp seal diets, 6 of which were present at pro- portions $>5 \%$ (Table 3 ). These included amphipods, Arctic cod, capelin, herring, sand lance and redfish. Individual harp seals are estimated to have consumed between 1 and 14 items, with a mean of $5.1 \pm 2.0$. We identified 26 prey items in hooded seal diets, 5 of which were present at proportions of $>5 \%$ and included amphipods, Atlantic argentine, capelin, euphausiids and redfish. Individuals are estimated to have consumed between 1 and 13 items, with a mean of $5.7 \pm 2.5$. Relative to adult hooded seals, adult harp seals consumed significantly greater percentages of amphipods, Arctic cod and sand lance. In turn, diets of adult hooded seals were comprised of higher percentages of Atlantic argentine and redfish relative to the diets of adult harp seals (Fig. 2a). Diets of juvenile hooded seals had significantly higher percentages of argentine, capelin, and euphausiids, while juvenile harp seal diets had higher percentages of sand lance (Fig. 2b). Other main effects are discussed under 'Results-intra-specific differ-

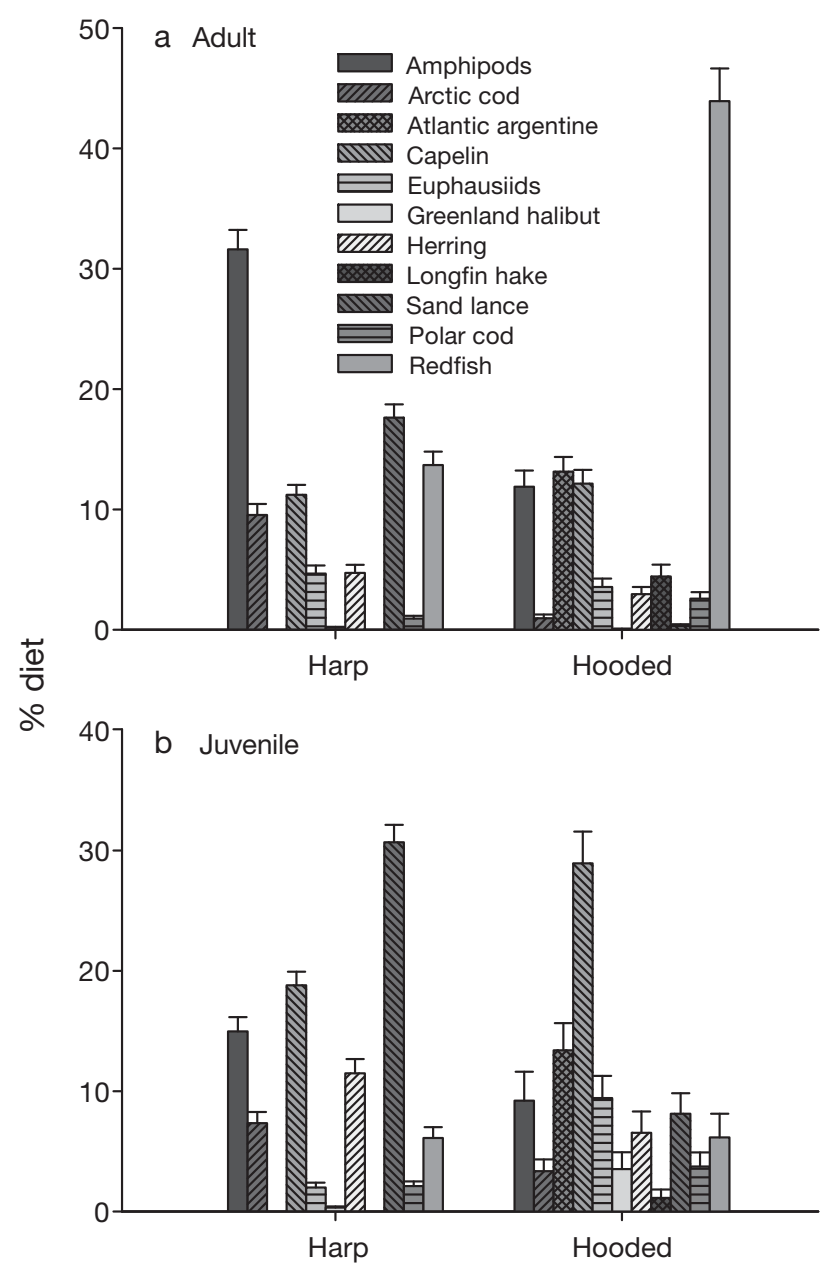

Fig. 2. Pagophilus groenlandicus and Cystophora cristata. Estimated principle diet components for (a) adults and (b) juveniles. Error bars represent SE 


\begin{tabular}{|c|c|c|c|c|c|}
\hline 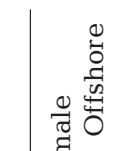 & 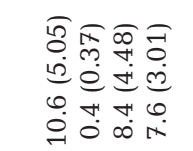 & 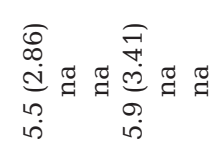 & 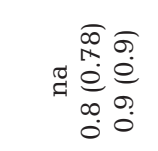 & 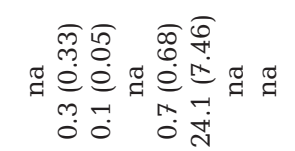 & 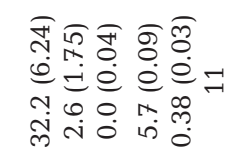 \\
\hline 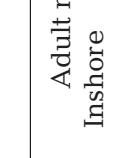 & 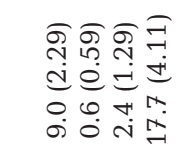 & 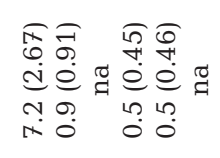 & 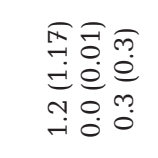 & 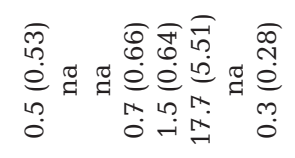 & 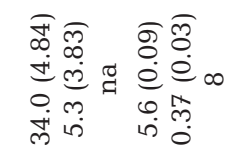 \\
\hline 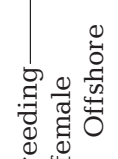 & 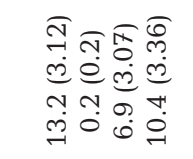 & 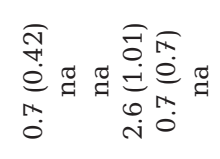 & 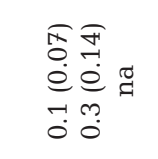 & 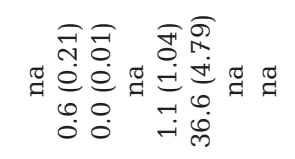 & 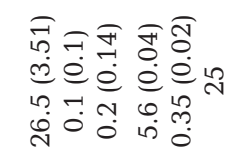 \\
\hline 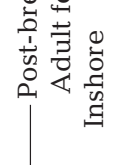 & 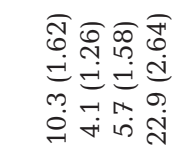 & 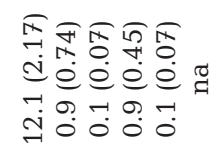 & 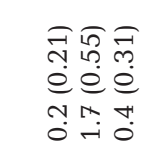 & 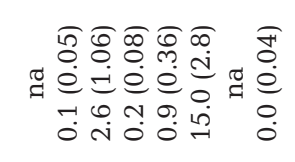 & 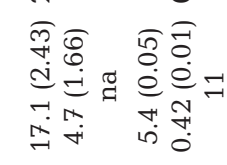 \\
\hline 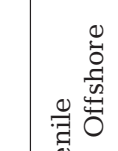 & 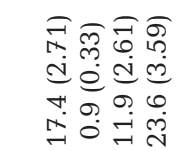 & 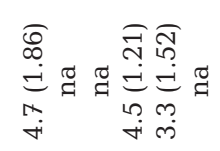 & 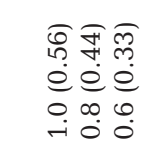 & 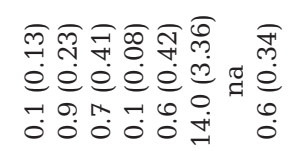 & 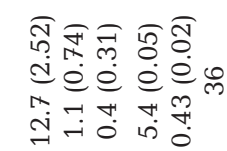 \\
\hline 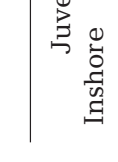 & 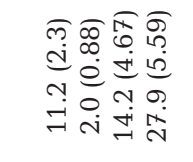 & 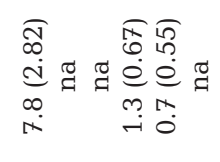 & 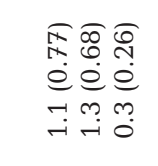 & 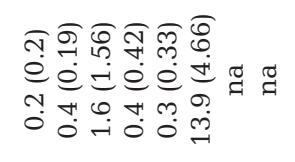 & 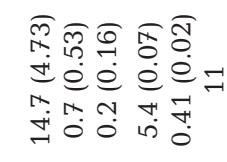 \\
\hline 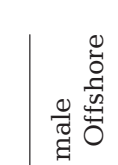 & 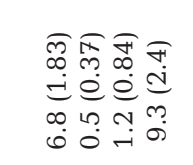 & 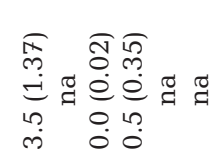 & 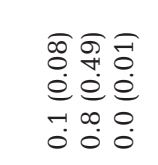 & 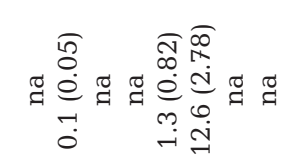 & 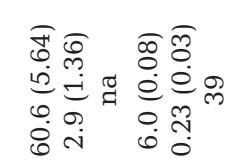 \\
\hline 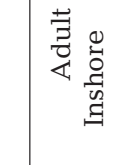 & 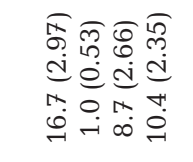 & 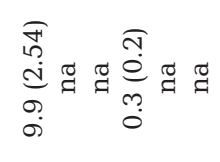 & 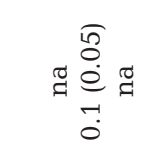 & 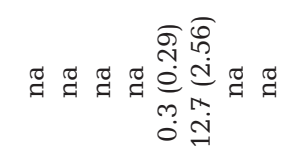 & 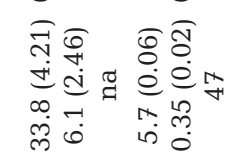 \\
\hline 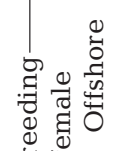 & 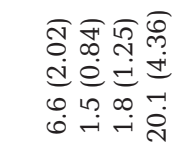 & 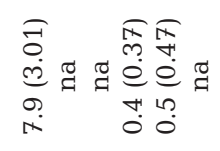 & 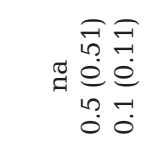 & 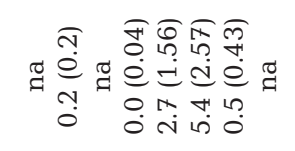 & 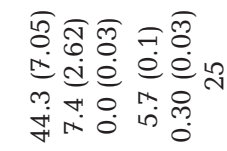 \\
\hline 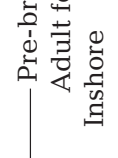 & 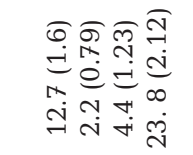 & 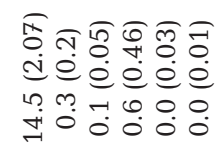 & 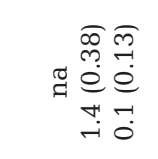 & 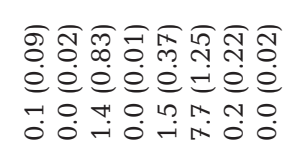 & 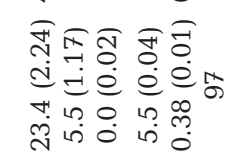 \\
\hline 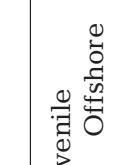 & 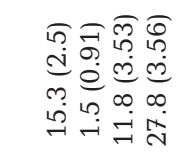 & 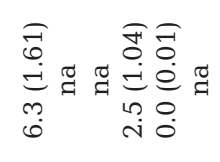 & 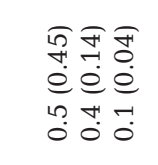 & 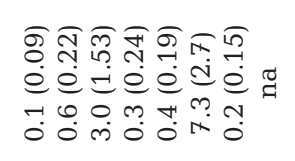 & 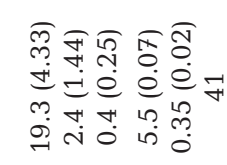 \\
\hline 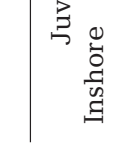 & 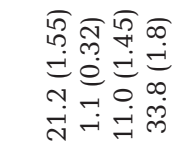 & 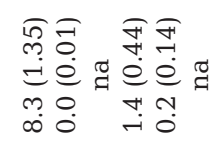 & 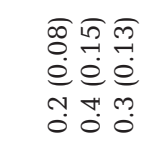 & 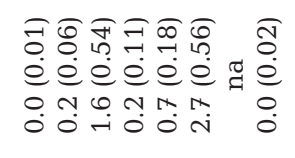 & 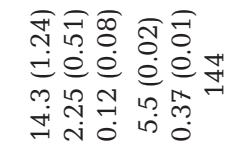 \\
\hline & 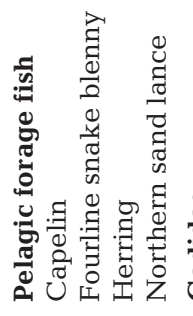 & 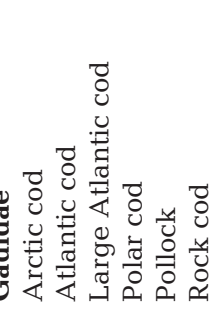 & 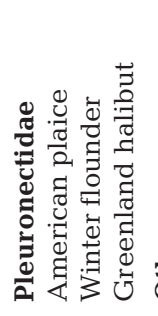 & 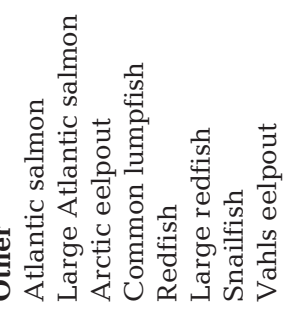 & 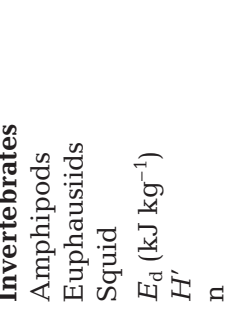 \\
\hline
\end{tabular}




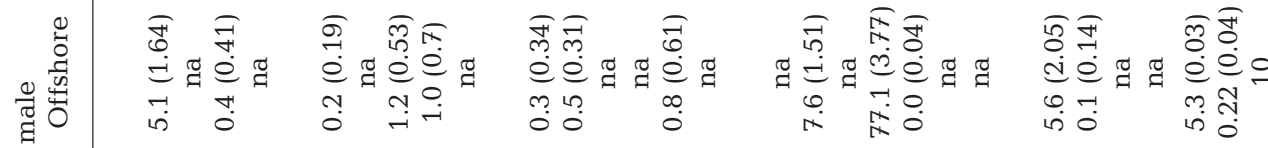

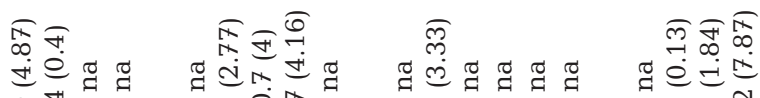

के

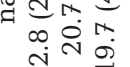

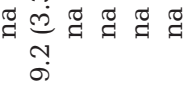

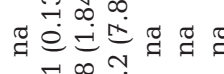

苁

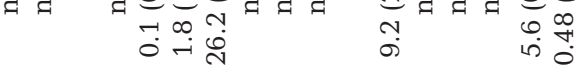

ॠ

ঙ্লে

ก)

需

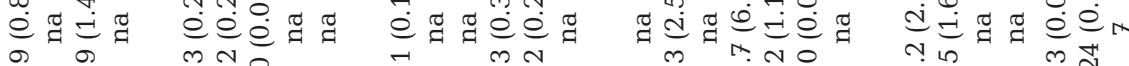

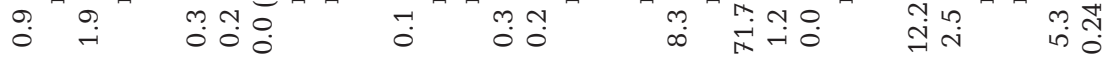

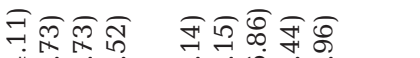

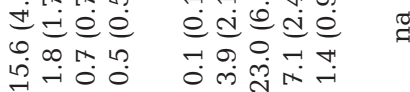

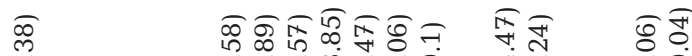

辛营

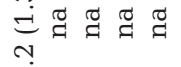

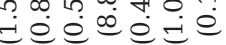

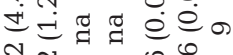

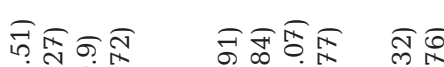

Hej व

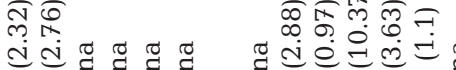

萑品

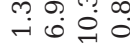

ii $\stackrel{\text { m }}{m}$

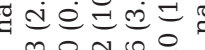

maid

ํ. ํ.

कळ

ปูi 00

(०) बृने

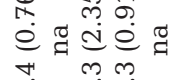

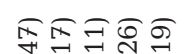

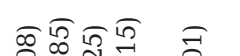

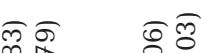

苛

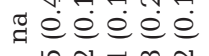

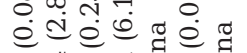

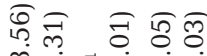

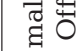

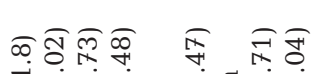

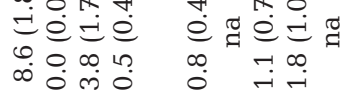

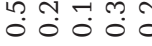

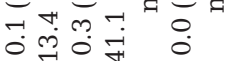

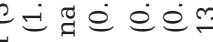

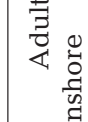

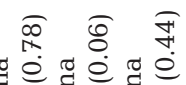

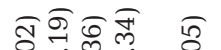

Aิ

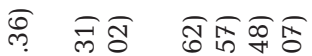

تृ

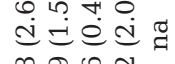

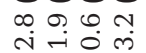

$\stackrel{m}{\rightarrow} \quad \overrightarrow{0} \infty$

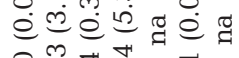

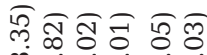

นึ่

ले

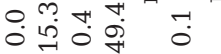

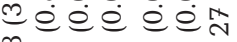

学 i

퓰

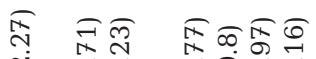

ปู่

ปี 6.

อ $9 \dot{9} \dot{0}$

고 옹

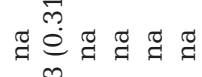

สิษ

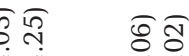

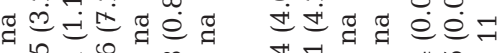

$\vec{\exists}=\dot{0}$

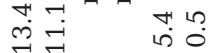

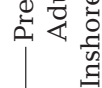

कृ

क्ष.

过过过

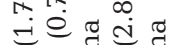

?

(

मुু

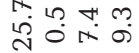

$\sum_{-1}^{-1} \int_{0}^{\pi} \int_{0}^{\pi} \pi$

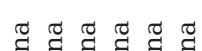

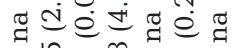

लூ

(⿻)

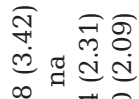

究 ॠ०

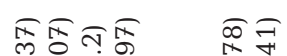

तิ 孚

तี่

의

मीं

苗

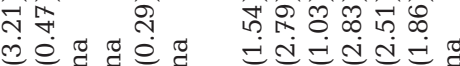

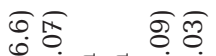

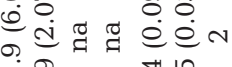

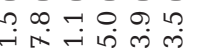

iो मां

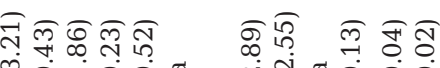

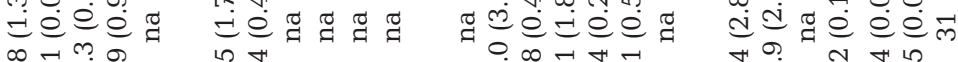

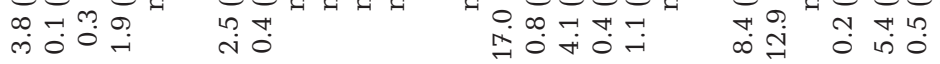

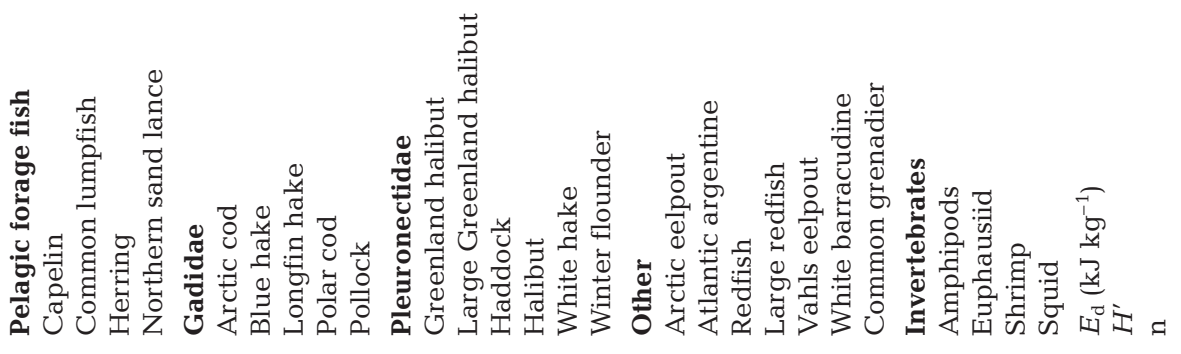


Table 5. Pagophilus groenlandicus (Erxleben, 1777) and Cystophora cristata (Erxleben, 1777). Morisita-Horn index $\left(C_{\mathrm{H}}\right)$ of dietary overlap. Degree of dietary overlap is small when $C_{\mathrm{H}}$ is between 0 and 0.29 , medium when $C_{\mathrm{H}}$ is between 0.30 and 0.59 and large when $C_{\mathrm{H}}>0.60$. na $=$ not applicable

\begin{tabular}{|llc|}
\hline Comparison & \multicolumn{1}{c|}{ Season } & $C_{\mathrm{H}}$ \\
\hline Harp & & \\
Adults & Pre- vs. Post-breeding & 0.91 \\
Juveniles & Pre- vs. Post-breeding & 0.94 \\
Adults vs. Juveniles & Pre-breeding & 0.79 \\
& Post-breeding & 0.89 \\
Hooded & & \\
Adults & Pre- vs. Post-breeding & 0.89 \\
Juveniles & Pre- vs. Post-breeding & na \\
Adults vs. Juveniles & Pre-breeding & 0.57 \\
& Post-breeding & na \\
Harp vs. hooded & & \\
Adults & Pre-breeding & 0.51 \\
& Post-breeding & 0.61 \\
Juveniles & Pre-breeding & 0.70 \\
& Post-breeding & na \\
\hline
\end{tabular}

ences'. Dietary overlap, as measured by $C_{\mathrm{H}}$, between harp seals and hooded seals was moderate for adults and high for juveniles (Table 5). $E_{\mathrm{d}}$ of harp seal diets was significantly higher than hooded seal diets $\left(F_{1,678}=\right.$ $16.78, \mathrm{p}<0.001)$. Conversely, hooded seals had marginally greater niche breadths $\left(F_{1,678}=4.0, \mathrm{p}=0.046\right)$.

\section{Intra-specific differences}

\section{Harp seals}

Dominant prey species in estimated diets differed by sex $(p=0.002)$, age class $(p<0.001)$, season $(p<0.001)$, area $(\mathrm{p}<0.001)$ and year $(\mathrm{p}<0.001)$ (Table 3$)$. Juveniles consumed a greater proportion of herring and northern sand lance while adults consumed higher percentages of amphipods, euphausiids and redfish. To control for age-class effects, we separated juveniles from adults in subsequent analyses.

Adults. In adults, both sexes consumed similarly high percentages of capelin and redfish (Figs. 3 \& 4); however, diet varied significantly between males and females $(p<0.001)$ in other components. Males consumed approximately twice the percentage of amphipods, while females consumed higher percentages of Arctic cod, snake blenny and sand lance.

There were significant seasonal ( $\mathrm{p}<0.001)$ differences in diet (Figs. $3 \& 4$ ) due primarily to higher percentages of amphipods in the pre-breeding period, while polar cod and redfish were higher in the postbreeding period. There was a significant effect of sampling area $(p<0.001)$ on estimated diets due primarily

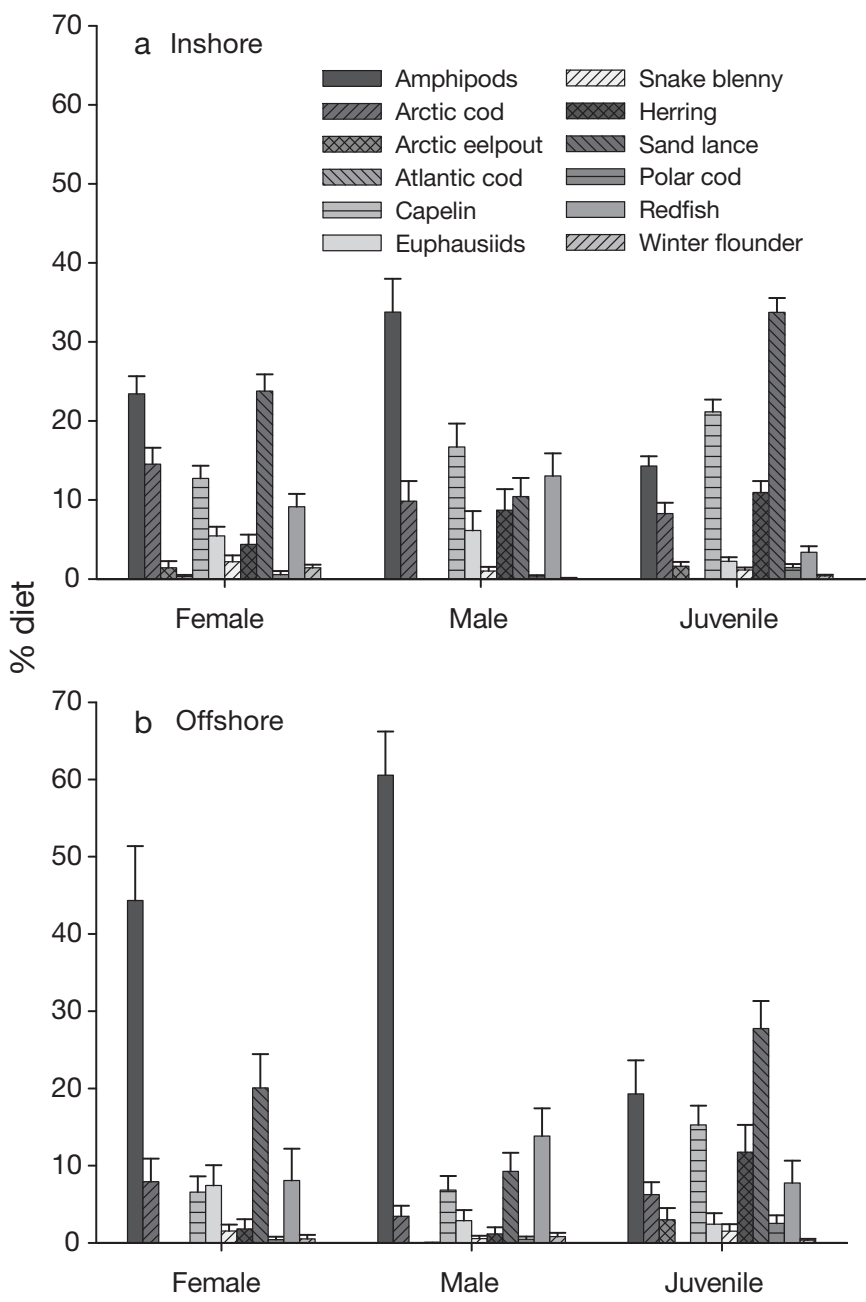

Fig. 3. Pagophilus groenlandicus. Estimated mean diet composition for adult females, adult males and juveniles from the pre-breeding period sampled in (a) inshore and (b) offshore areas. Error bars represent SE

to higher percentages of amphipods and redfish consumed in the offshore, while higher percentages of pelagic forage fish, namely Arctic cod, capelin and sand lance, were consumed in the inshore. There was also a significant interaction between area and season $(p<0.001)$. The inshore-offshore differences remained generally consistent within seasons; however, there were significant differences between seasons for both inshore $(p=0.005)$ and offshore samples $(p<0.001)$. Inshore, the percentage of redfish in the diet was higher in the post-breeding period. Offshore, amphipods were almost double their pre-breeding level, while herring, polar cod and redfish were consumed in greater percentages in the post-breeding period.

Diet composition varied significantly by year ( $p<$ 0.001). Post hoc analysis showed that the diets in both 1994 and 1995 differed from all other years (Fig. 5), 
while 1996, 2000, 2002 and 2004 were all similar to one another (all p > 0.14); 1998 differed from 2002 ( $\mathrm{p}=$ $0.036)$ and $2004(p=0.027)$. In 1994, an elevated percentage of amphipods and decreased percentages of capelin and sand lance were seen relative to other years. Similarly, in 1995 there were elevated percentages of Arctic cod and lower levels of capelin. Increased percentages of American plaice, Atlantic cod, lumpfish and Greenland halibut Reinhardtius hippoglossoides were estimated in the 1998 diet.

Juveniles. There were no significant differences among juvenile harp seals of different sexes $(p=0.54)$, although diets did vary by season, year and area (all $\mathrm{p}<$ 0.001). Seasonal variation in diet composition was primarily due to elevated levels of sand lance in the prebreeding period, and elevated levels of redfish in the post-breeding period. Offshore diets were characterised by a higher proportion of redfish and polar cod, while inshore diets had higher proportions of sand lance.

A similar pattern to adults was observed in annual differences for juveniles. Post hoc analysis indicated

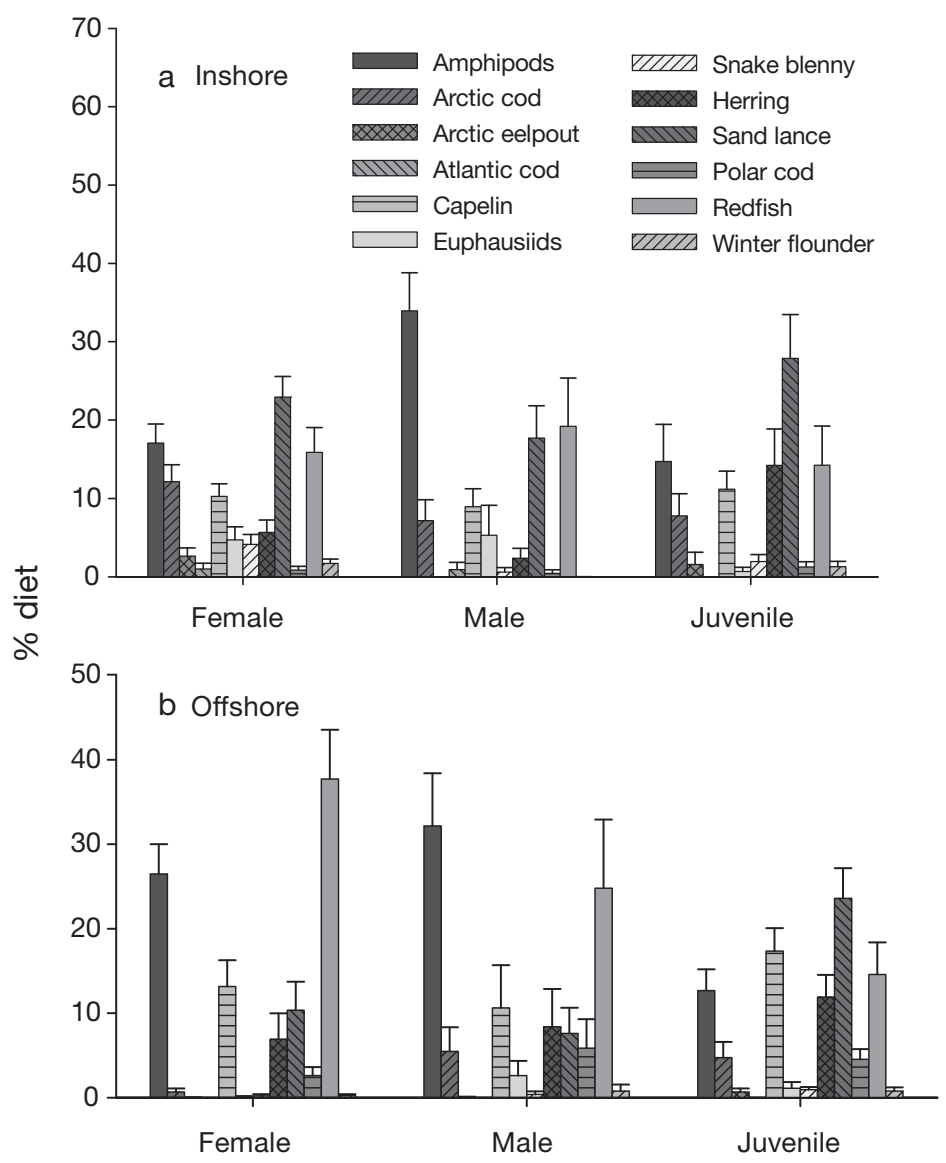

Fig. 4. Pagophilus groenlandicus. Estimated mean diet composition for adult females, adult males and juveniles from the post-breeding period sampled in (a) inshore and (b) offshore areas. Error bars represent SE that 1994 differed from all years except 1998, while 1995 in turn differed from all other years. With the exception of differences between 1996 and 2004 ( $\mathrm{p}=$ 0.013), diet composition was similar from 1996 to 2004. In 1994, there were elevated percentages of amphipods and decreased percentages of capelin and redfish relative to other years. Similarly, in 1995 there were higher percentages of Arctic cod and sand lance and lower levels of capelin in the diet.

$E_{\mathrm{d}}$ and $H^{\prime}$. Mean $E_{\mathrm{d}}$ of harp seal diets ranged between 5.4 and $6.0 \mathrm{~kJ} \mathrm{~g}^{-1}$ (Table 3) and although differences in energy density were generally small, there was a significant effect of $\operatorname{sex}\left(F_{1,525}=25.95, \mathrm{p}<0.001\right)$, age class $\left(F_{1,525}=31.11, \mathrm{p}<0.001\right)$, area $\left(F_{1,525}=28.06\right.$, $\mathrm{p}<0.001)$ and season $\left(F_{1,525}=16.13, \mathrm{p}<0.001\right)$. In adults, which had higher $E_{\mathrm{d}}$ overall, $E_{\mathrm{d}}$ was significantly greater in males than females $\left(F_{1,293}=4.75, \mathrm{p}=\right.$ 0.033). For both adults and juveniles, offshore diets had higher $E_{\mathrm{d}}$ and the $E_{\mathrm{d}}$ was higher in the prebreeding period.

Mean $H^{\prime}$ for harp seals ranged between 0.23 and 0.43 (Table 3 ) and there were significant effects of age class $\left(F_{1,525}=13.76, \mathrm{p}<0.001\right)$, season $\left(F_{1,525}=36.59\right.$, $\mathrm{p}<0.001)$ and area $\left(F_{1,525}=28.06, \mathrm{p}<0.001\right)$. In adults, females had a significantly higher $H^{\prime}$ than males $\left(F_{1,525}=8.1, \mathrm{p}=0.005\right) . H^{\prime}$ values were all higher in juveniles, in the offshore, and in post-breeding diets.

\section{Hooded seals}

The dominant prey species in hooded seal diets differed by sex $(p=0.04)$, age class $(p<0.001)$, season $(p<0.001)$ and area $(p<0.001)$. Diets for adults had higher percentages of redfish, while juveniles consumed higher percentages of Arctic cod, herring and sand lance (Fig. 6). Again, we separated adults from juveniles to control for age-class effects. There were no sex differences between male and female juvenile hooded seals $(p=0.55)$. Sample sizes for juveniles were too small to examine other main effects.

Adults. We found significant differences in diets of males and females $(p=0.011$, with males consuming significantly greater percentages of redfish and Greenland halibut and females consuming greater percentages of blue hake and white baraccudine. There was a significant effect of season on diet composition $(\mathrm{p}<$ 0.001). Differences were due primarily to higher percentages of argentine and capelin in the pre-breeding period, while the percentage of redfish was 2-fold higher in the post-breeding period. Animals sampled in Greenland varied significantly from the other 2 areas for many species $(\mathrm{p}<0.001)$, but differences were primarily due to elevated percentages of longfin hake and polar cod and decreased levels of argentine. There 

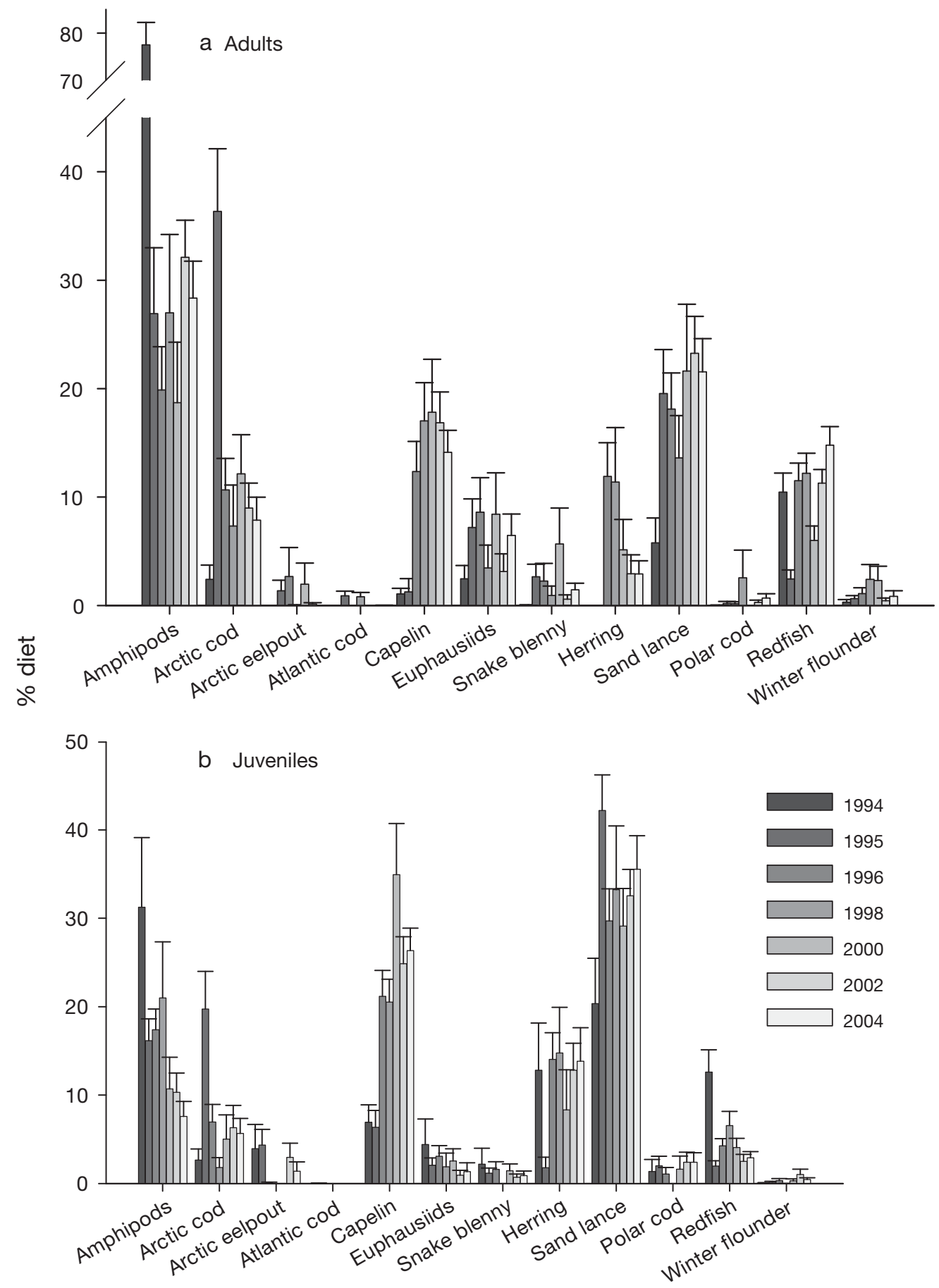

Fig. 5. Pagophilus groenlandicus. Estimated mean diet composition for (a) adults and (b) juveniles from the pre-breeding period in different years (1994 to 2004). Error bars represent SE

were significant differences between inshore and offshore samples with respect to the percentage of capelin (higher in inshore) and redfish (higher in offshore).

$E_{\mathrm{d}}$ and $H^{\prime}$. Mean $E_{\mathrm{d}}$ of hooded seal diets ranged between 5.3 and $5.6 \mathrm{~kJ} \mathrm{~g}^{-1}$ (Table 4), but varied with season $\left(F_{1,152}=5.90, \mathrm{p}<0.016\right)$ where $E_{\mathrm{d}}$ was higher in pre-breeding, and with area $\left(F_{1,152}=12.49, \mathrm{p}<0.001\right)$.
There were no significant differences in $E_{\mathrm{d}}$ between juveniles and adults $\left(F_{1,152}=0.04, \mathrm{p}=0.84\right)$ or between males and females $\left(F_{1,152}=0.053, \mathrm{p}=0.82\right)$.

Mean $H^{\prime}$ for hooded seals ranged between 0.22 and 0.50 (Table 4), with evidence for significant effects of season $\left(F_{1,152}=18.37, \mathrm{p}<0.001\right)$, age class $\left(F_{1,152}=\right.$ $14.95, \mathrm{p}<0.001)$ and area $\left(F_{1,525}=14.24, \mathrm{p}<0.001\right) . H^{\prime}$ 


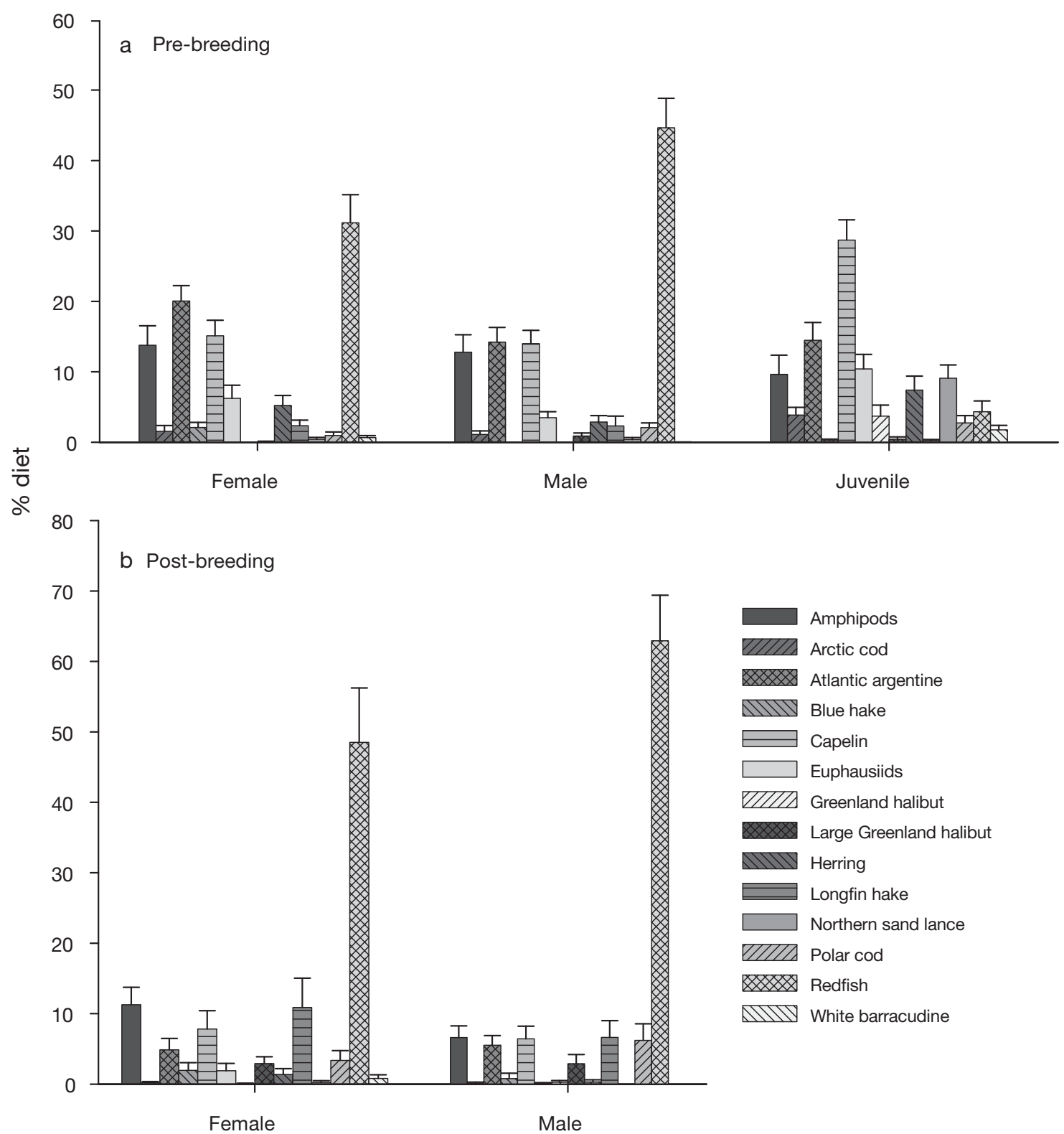

Fig. 6. Cystophora cristata. Estimated mean diet composition for adult females, adult males and juveniles from the (a) pre- and (b) post-breeding periods. Error bars represent SE

was higher in both juveniles and in the pre-breeding season, as well as greater in the offshore. There was no significant difference between adult males and females $\left(F_{1,152}=2.69, \mathrm{p}=0.10\right)$.

\section{DISCUSSION}

\section{Species differences}

Our results show that species differences in diets appear to reflect previously defined differences in diving behaviour and distribution (Folkow et al. 1996, 2004,
Folkow \& Blix 1999), in that harp seals had higher percentages of pelagic prey whereas hooded seals had higher percentages of demersal prey. Furthermore, while our results agree generally with estimates of stomach contents of the species consumed, there are important differences, perhaps most important of which is the high proportion of amphipods in both harp and hooded seal diets. Amphipods have been reported in high frequencies in stomach contents of both seal species (e.g. Sergeant 1991, Lawson et al. 1995, Haug et al. 2007); however, it has always been difficult to quantitatively assess their contribution to the overall diet. The large contribution of Atlantic argentine to hooded seal diets was 
also unexpected based on previous studies. Information on argentine is scarce, although they can be abundant at the continental shelf edge, likely in aggregations, and at depths of 140 to 1440 m (Froese \& Pauly 2007), which are well within the diving and foraging range for these predators (Folkow \& Blix 1999).

Dietary overlap between harp and hooded seals was moderate for adults and high for juveniles (Table 5). However, given what is known about the differences in foraging behaviour and distribution of these 2 species (Folkow et al. 1996, 2004, Folkow \& Blix 1999), it is difficult to assess how this overlap may be manifested over temporal and spatial scales. Recent data from the northwest Atlantic (Stenson \& Sjare 1997, G. B. Stenson unpubl. data) indicate that they are spatially separated for much of the time, with harp seals being found on the continental shelf while hooded seals inhabit the shelf edge, although they may co-occur occasionally outside of the breeding period (Folkow et al. 1996, 2004). Greenland Sea stomach-content data suggest that although both species co-occurred, diets varied significantly between the species (Haug et al. 2004). Specifically, the relative contribution to the diet of pelagic amphipods, Arctic cod, capelin and squid varied between harp and hooded seals. It is likely that differences in prey selection resulted from hooded seals diving to deeper depths than harp seals (Folkow \& Blix 1999, Folkow et al. 2004, Haug et al. 2004).

Although $E_{\mathrm{d}}$ was higher in harp seal diets because of greater percentages of pelagic forage fish and invertebrates, hooded seals had larger niche breadths. This is similar to sympatric terrestrial carnivores, where larger species tend to have larger niche breadths, although the diets of smaller predators are often nested within those of larger predators (i.e. Radloff \& du Toit 2004), resulting in a fairly large degree of dietary overlap.

\section{Age-class effects}

Although harp seal diets were dominated by pelagic species and significant dietary overlap was evident in juveniles and adults, juveniles consumed more pelagic forage fish, whereas adults consumed more amphipods and redfish. Ontogenetic diet shifts have been demonstrated in harp seals through the analysis of stomach contents, where the proportion of pelagic forage fish declines as the proportion of pelagic invertebrates increases in the diet as seals grow older (Lawson et al. 1995). In hooded seals, adult diets were dominated by redfish, which comprised only a small portion of the diet for juveniles. However, percentages of amphipods and argentine were equivalent. Similar to juvenile harp seals, juvenile hooded seals consumed more pelagic forage fish than adults.
Ontogenetic diet differences have been noted for many animal taxa and these are thought to result from either behavioural development or differing energetic costs associated with body size (reviewed in Bolnick et al. 2003). In pinnipeds, juveniles undergo a period of physiological and behavioural development related to dive capacity (e.g. Noren et al. 2005). For both harp and hooded seals, niche breadth was higher in juveniles, which may indicate more experimentation, or at least less specialisation, by these inexperienced foragers. In harp seals, $E_{\mathrm{d}}$ was higher in adults, potentially mandated by overall higher energy costs related to maturation and body size. Similar results have been found between juvenile and adult grey seals (Beck et al. 2007). In hooded seals, $E_{\mathrm{d}}$ was equivalent between juveniles and adults. However, these differences or equivalencies in consumed energy may only be apparent, as metabolisable energy from the diet can vary with prey type.

Diet differences between adults and juveniles could also reflect differences in distribution (Sergeant 1991), resulting in differences in prey availability and prey selection (e.g. Field et al. 2005). Amphipods have high lipid content (the present study: mean $8.4 \%$, range 3.0 to $19.5 \%$ ) and foraging and handling costs are presumably small if animals are feeding within dense aggregations of these zooplankton (Costa et al. 1989). However, energy assimilation from crustaceans is lower than from fish (Keiver et al. 1984, Martensson et al. 1994). Although the energy return is great with respect to lipid content, efficient high consumption of amphipods may require larger gut capacity to process high volumes of indigestible chitinous material, as assimilation efficiencies are lower (e.g. Lawson et al. 1997). It has been hypothesised that efficient consumption of benthic forage fish may also require larger gut capacity (Beck et al. 2007, Tucker et al. 2007). In captive feeding studies in seals, the assimilation efficiency of gross energy intake is between 10 and $23 \%$ lower for crustaceans than for pelagic forage fish (Keiver et al. 1984, Martensson et al. 1994). Studies have also shown that the assimilation efficiency of gross energy can be lower for benthic fish species relative to pelagic forage fish (e.g. Lawson et al. 1997). In captivity, harp seal pups are unable to maintain body mass while on an exclusively crustacean diet (Martensson et al. 1994). Therefore juvenile harp seals in the wild may consume more fish in order to sustain high costs associated with growth. Similarly, juvenile hooded seals may select fish species with a higher assimilation efficiency to maximise net gains. Thus ontogenetic diet shifts in seals likely entail complex tradeoffs between distribution, behavioural and physiological development, foraging costs and the efficient digestion of prey. 


\section{Sex differences}

We found significant differences in diet between adult males and females in both species. Although preliminary analyses of telemetry studies have not revealed any differences in movement or diving behaviour between male and female harp seals (G. B. Stenson unpubl. data), sex-specific differences in mean dive depths in the post-breeding period has been found in hooded seals (Bajzak et al. 2009). Sex differences in foraging behaviour of pinnipeds may reflect differences in sex-specific costs of reproduction, body size or competitive abilities (i.e. Beck et al. 2003, 2007). Thus sex differences in diets in adult harp seals in the pre-breeding period may be related to sex-specific costs associated with pregnancy, lactation and reproduction. We found that $E_{\mathrm{d}}$ was higher in male than in female harp seals due to the higher proportion of amphipods. However, the available energy in diets of males is likely lower due to lower assimilation efficiencies associated with consumption of crustaceans. Indeed, females may not be able to efficiently meet the high energetic costs associated with pregnancy on a predominantly amphipod diet despite potentially lower foraging costs. This could also be the case for adult hooded seals, as the similarity in $E_{\mathrm{d}}$ between males and females may only be apparent due to potentially lower assimilation efficiencies of the predominantly redfish diet of males. Although differences in sex-specific costs of reproduction may contribute to the estimated differences in the diets of males and females, the costs associated with maintaining larger body size in males may be a more significant factor in this species. The lack of sex differences in the diets of juveniles is probably indicative of the lack of reproductive costs or, in the case of hoods at that age, lack of significant body-size dimorphism.

\section{Spatial and temporal differences}

In both adult and juvenile harp seals, we found inshore-offshore differences in diets based on QFASA. These were due to higher percentages of amphipods and redfish in the offshore and higher percentages of pelagic fish (capelin, herring and Arctic cod) in inshore diets. These results are supported by data from stomach-content analyses that have previously indicated differences in inshore and offshore diets for harp seals (Lawson et al. 1995, 1998, Lawson \& Stenson 1997). Inshore, adult diets tended to be dominated by capelin, herring, sand lance and Arctic cod, while offshore, diets tended to be dominated by invertebrates, capelin, sand lance and a mix of demersal species. Although sample sizes were small, we found large-scale regional differences in hooded seal diets as well, confirming general conclusions from stomach-content analysis (e.g. Hammill \& Stenson 2000, Haug et al. 2007) and suggesting that spatial effects are an important component of hooded seal diet breadth.

There were significant differences between pre- and post-breeding diets for both harp and hooded seals which resulted in higher $E_{\mathrm{d}}$ overall in the pre-breeding period. In adult harp seals, this was due to a higher percentage of energy-rich amphipods in the prebreeding period, whereas polar cod and large redfish were higher in the post-breeding period. Polar cod, which are also bathypelagic like redfish, are generally found at higher latitudes, associated with ice, and found mainly in offshore waters at or beyond the edge of the continental shelf (Froese \& Pauly 2007). Therefore, increased consumption may be linked to postbreeding/pre-moulting feeding in proximity to 'the Front'. In juvenile harp seals, seasonal variation in diet composition was primarily due to elevated levels of sand lance in the pre-breeding period, and again elevated levels of redfish in the post-breeding period. Differences in hooded seal diets were due primarily to higher proportions of argentine and capelin in the prebreeding period, while the proportion of large redfish was higher in the post-breeding period.

Inter-annual variation in pinniped diets is assumed to reflect variation in prey abundance and subsequent encounter rates (Bowen \& Siniff 1999). Thus interannual variation in harp seal diets may reflect large ecological changes that occurred in the northwest Atlantic during the last decade (e.g. Lilly \& Simpson 2000, Carscadden et al. 2001, Rose 2004). Capelin declined in northern areas in the early 1990s (Carscadden et al. 2001, Rose 2004) becoming scarce along the coast of Labrador and the Grand Banks. Coincidentally, abundance of capelin increased on the Flemish Cap and the Scotian Shelf (Lilly \& Simpson 2000, Carscadden et al. 2001). Concurrently, Arctic cod distribution shifted southward from Labrador to coastal Newfoundland and the Grand Banks out to the shelf edge. This expansion in distribution and Arctic cod biomass peaked in 1995 (Lilly \& Simpson 2000). By 1998-1999, Arctic cod appeared to be returning to northerly distributions (Lilly \& Simpson 2000); however, by 2000 capelin had not returned to waters off the Labrador coast (Department of Fisheries and Oceans Canada 2001). Concurrent with these observations, our QFASA results indicate that in 1994 there were decreased proportions of capelin and sand lance in the diet relative to other years. Similarly, in 1995 there were higher percentages of Arctic cod and a lower percentage of capelin. Indeed, during the 1990s there were notable changes in harp seal distribution and diet as indicated from stomach-content analysis. From 1990 to 
1995, Arctic cod were a significant prey species found in seals' stomachs in inshore areas in the spring (Lawson et al. 1995). Arctic cod had been the key prey species in winter diets since 1986 (Stenson \& Perry 2001). Anecdotal reports have suggested an increase in harp seal abundance in inshore waters over the 1990s (Lacoste \& Stenson 2000, Sjare et al. 2004), although some portion of the population was continuing to consume capelin in offshore areas (Stenson \& Perry 2001). In addition, since the late 1990s, the amount of sand lance has increased in offshore diets estimated from stomach contents, while herring has increased in inshore diets. Furthermore, changes in diets of other species, most notably seabirds, corroborate other sources of information indicating major changes in prey abundances (Montevecchi \& Myers 1996, Davoren \& Montevecchi 2003).

In the present study, we used an averaging procedure of modelling iterations to incorporate uncertainty in CCs. The assimilation, deposition and estimates of CCs for individual FAs have now been established for 5 species of phocids (including harp seals) and otariid seals, as well as for mink and seabirds, through controlled feeding studies (reviewed in Iverson 2009). While it is true that no hooded seals were included in the 5 species, we are confident in our application of the QFASA model to this species given that CCs are all remarkably similar amongst species and taxa (i.e. pathways of FA metabolism are conserved). However, the magnitude of individual CCs can vary between species. When applied in QFASA, these can result in differences in the proportional contribution of diet components but not the species composition per se (Tucker 2007). We averaged CCs from 2 related phocid species (harp and grey seals) to at least partially account for any uncertainty in this parameter. Further research will no doubt improve the application of FAs and CCs to estimating predator diets.

We have shown that inter-and intra-specific variation in diet of these species, whether due to sex or ontogenetic differences, are also influenced by geographic and temporal effects (seasonal and interannual). Thus, these sources of variation will need to be considered when determining the role of these uppertrophic level predators in marine ecosystems. Segregation in diet (ontogenetic or sex) may be influenced both by intrinsic energy requirements (growth or pregnancy) and the energetic properties associated with that diet (foraging costs and assimilation efficiency). Essentially, juveniles and females of both species may require high-quality prey (i.e. pelagic forage fish) to meet high energy demands, while males are able to process higher proportions of lower-quality prey (i.e. amphipods, benthic fish), offsetting lower returns from that diet (lower energy assimilation efficiencies) due to lower energetic demands. From an ecological perspective, this capacity or requirement to segregate diets would also have the benefit of reducing intra-specific competition.

Acknowledgements. We thank D. McKinnon, W. Penney and D. Wakeham (Department of Fisheries and Oceans, St. John's, Newfoundland) for collecting and processing seal samples, and for age analysis. We thank the many seal hunters and fishermen who have contributed to the biological sampling programme over the years. We also thank the crew and helicopter crew of the CCGS 'Anne-Harvey', March 2004 and 2005. We thank David Hardie for providing supplementary fish samples from deep-water trawl surveys of the Davis strait. M. Trudel provided assistance with PBS mapping. This study was supported by research and equipment grants to S.J.I. from the Natural Sciences and Engineering Research Council (NSERC) Canada, by the Department of Fisheries and Oceans Canada, and by a Quebec Graduate Fellowship (FCAR) to S.T.

\section{LITERATURE CITED}

Bajzak CE, Côté SD, Hammill MO, Stenson G (2009) Intersexual differences in the postbreeding foraging behaviour of the Northwest Atlantic hooded seal. Mar Ecol Prog Ser (in press)

Beck CA, Bowen WD, McMillan JI, Iverson SJ (2003) Sex differences in the diving behaviour of a size-dimorphic capital breeder: the grey seal. Anim Behav 66:777-789

Beck CA, Iverson SJ, Bowen WD (2005) Blubber fatty acids of gray seals reveal sex differences in the diet of a sizedimorphic marine carnivore. Can J Zool 83:377-388

Beck CA, Iverson SJ, Bowen WD, Blanchard W (2007) Sex differences in grey seal diet reflect seasonal variation in foraging behaviour and reproductive expenditure: evidence from quantitative fatty acid signature analysis. J Anim Ecol 76:490-502

Bolnick DI, Svanback R, Fordyce JA, Yang LH, Davis JM, Hulsey CD, Forister ML (2003) The ecology of individuals: incidence and implications of individual specialization. Am Nat 161:1-28

Bowen WD, Siniff DB (1999) Distribution, population biology, and feeding ecology of marine mammals. In: Renolds JE III, Rommel SA (eds) Biology of marine mammals. Smithsonian Institution Press, Washington, DC, p 423-484

> Bowen WD, Sergeant DE, Oritsland T (1983) Validation of age determination in the harp seal, Phoca groenlandica, using dentinal annuli. Can J Fish Aquat Sci 40:1430-1441

Breed GA, Bowen WD, McMillan JI, Leonard ML (2006) Sexual segregation of seasonal foraging habitats in a nonmigratory marine mammal. Proc R Soc Lond B Biol Sci 273: 2319-2326

> Budge SM, Iverson SJ, Bowen WD, Ackman RG (2002) Among- and within-species variability in fatty acid signatures of marine fish and invertebrates on the Scotian Shelf, Georges Bank, and southern Gulf of St. Lawrence. Can J Fish Aquat Sci 59:886-898

Carscadden JE, Frank KT, Leggett WC (2001) Ecosystem changes and the effects on capelin (Mallotus villosus), a major forage species. Can J Fish Aquat Sci 58:73-85

Chabot D, Leblanc MJ, Stenson GB, Kapel FO, Audet C (2006) Growth and condition of hooded seal (Cystophora cristata): seasonal and density effects. Joint ICES/NAFO 
Working Group on Harp and Hooded Seals. ICES HQ WPSEA-161, Copenhagen

Cooper MH (2004) Fatty acid metabolism in marine carnivores: implications for quantitative estimation of predator diets. PhD thesis, Dalhousie University, Halifax

Costa DP, Croxall JP, Duck CD (1989) Foraging energetics of Antarctic fur seals in relation to changes in prey availability. Ecology 70:596-606

Davoren GK, Montevecchi WA (2003) Signals from seabirds indicate changing biology of capelin stocks. Mar Ecol Prog Ser 258:253-261

Department of Fisheries and Oceans Canada (2001) Capelin in Subarea $2+$ Div. 3KL Update. DFO Science Stock Status Report B2-02

Efron B, Tibshirani RJ (1998) An introduction to the bootstrap. Chapman and Hall/CRC, Boca Raton, FL

Field IC, Bradshaw CJA, Burton HR, Sumner MD, Hindell MA (2005) Resource partitioning through oceanic segregation of foraging juvenile southern elephant seals. Oecologia 142:127-135

Folch J, Lees M, Sloane-Stanly GH (1957) A simple method for the isolation and purification of total lipids from animal tissues. J Biol Chem 226:497-509

Folkow LP, Blix AS (1999) Diving behaviour of hooded seals (Cystophora cristata) in the Greenland and Norwegian Seas. Polar Biol 22:61-74

Folkow LP, Martensson P, Blix AS (1996) Annual distribution of hooded seals (Cystophora cristata) in the Greenland and Norwegian Seas. Polar Biol 16:179-189

Folkow LP, Nordøy ES, Blix AS (2004) Distribution and diving behaviour of harp seals (Pagophilus groenlandicus) from the Greenland Sea stock. Polar Biol 27:281-298

Froese R, Pauly D (eds) (2007) FishBase. www.fishbase.org

Hammill MO, Stenson GB (2000) Estimated prey consumption by harp seals (Phoca groenlandica), hooded seals (Cystophora cristata), grey seals, (Halichoerus grypus) and harbour seals (Phoca vitulina) in Atlantic Canada. J Northwest Atl Fish Sci 26:1-23

- Hammill MO, Kingsley MCS, Beck GG, Smith TG (1995) Growth and condition in the Northwest Atlantic harp seal. Can J Fish Aquat Sci 52:478-488

Haug T, Nilssen KT, Lindblom L (2004) Feeding habits of harp and hooded seals in drift ice waters along the east coast of Greenland in summer and winter. Polar Res 23:35-42

Haug T, Nilssen KT, Lindblom L, Lindstrøm U (2007) Diets of hooded seals in coastal and drift ice waters along the east coast of Greenland. Mar Biol Res 3:123-133

Iverson SJ (1993) Milk secretion in marine mammals in relation to foraging: can milk fatty acids predict diet? Symp Zool Soc Lond 66:263-291

Iverson SJ (2009) Tracing aquatic food webs using fatty acids: from qualitative indicators to quantitative determination. In: Arts MT, Brett MT, Kainz M (eds) Lipids in aquatic ecosystems. Springer-Verlag, New York (in press)

- Iverson SJ, Frost KJ, Lowry LF (1997) Fatty acid signatures reveal fine scale structure of foraging distribution of harbor seals and their prey in Prince William Sound, Alaska. Mar Ecol Prog Ser 151:255-271

Iverson SJ, Lang SL, Cooper MH (2001) Comparison of the Bligh and Dyer and Folch methods for total lipid determination in a broad range of marine tissue. Lipids 36: $1283-1287$

Iverson SJ, Field C, Bowen WD, Blanchard W (2004) Quantitative fatty acid signature analysis: a new method of estimating predator diets. Ecol Monogr 74:211-235

- Iverson SJ, Springer AM, Kitaysky AS (2007) Seabirds as indicators of food web structure and ecosystem variability: qualitative and quantitative diet analyses using fatty acids. Mar Ecol Prog Ser 352:235-244

Kapel FO (1995) Feeding ecology of harp and hooded seals in the Davis Strait-Baffin Bay region. In: Blix AS, Walløe L, Ultang $\varnothing$ (eds) Whales, seals, fish and man. Elsevier Science B.V., Amsterdam

Keiver KM, Ronald K, Beamish FWH (1984) Metabolizable energy requirements for maintenance and faecal and urinary losses of juvenile harp seals (Phoca groenlandica). Can J Zool 62:769-776

Krebs CJ (1999) Ecological methodology. Harper Collins, New York

Lacoste KN, Stenson GB (2000) Winter distribution of harp seals (Phoca groenlandica) off eastern Newfoundland and southern Labrador. Polar Biol 23:805-811

> Lawson JW, Stenson GB (1997) Diet of Northwest Atlantic harp seals (Phoca groenlandica) in offshore areas. Can J Zool 75:2095-2106

Lawson JW, Stenson GB, McKinnon DG (1995) Diet of harp seals (Phoca groenlandica) in nearshore waters of the northwest Atlantic during 1990-1993. Can J Zool 73: $1805-1818$

- Lawson JW, Hare JA, Noseworthy E, Friel JK (1997) Assimilation efficiency of captive ringed seals (Phoca hispida) fed different diets. Polar Biol 18:107-111

Lawson JW, Anderson JT, Dalley EL, Stenson GB (1998) Selective foraging by harp seals Phoca groenlandica in nearshore and offshore waters of Newfoundland, 1993 and 1994. Mar Ecol Prog Ser 163:1-10

Lewis S, Benvenuti S, Dall'Antonia L, Griffiths R, Money L, Sherratt TN, Wanless S, Hamer KC (2002) Sex-specific foraging behaviour in a monomorphic seabird. Proc R Soc Lond B 269:1687-1693

Lilly GR, Simpson M (2000) Distribution and biomass of capelin, Arctic cod and sand lance on the Northeast Newfoundland Shelf and Grand Bank as deduced from bottom-trawl surveys. DFO Can Stock Assess Sec Res Doc 2000/091

Martensson PE, Nordoy ES, Blix AS (1994) Digestibility of crustaceans and capelin in harp seals (Phoca groenlandica). Mar Mamm Sci 10:325-331

Montevecchi WA, Myers RA (1996) Dietary changes of seabirds reflect changes in pelagic food webs. Sarsia 80: 313-322

> Nordstrom CA, Wilson LJ, Iverson SJ, Tollit DJ (2008) Evaluating quantitative fatty acid signature analysis (QFASA) using harbour seals Phoca vitulina richardsi in captive feeding studies. Mar Ecol Prog Ser 360:245-263

Noren SR, Iverson SJ, Boness DJ (2005) Development of the blood and muscle oxygen stores in gray seals (Halichoerus grypus): implications for juvenile diving capacity and the necessity of a terrestrial postweaning fast. Physiol Biochem Zool 78:482-490

> Polis GA (1984) Age structure component of niche width and intraspecific resource partitioning: Can age groups function as ecological species? Am Nat 123:541-564

Potelov V, Nilssen KT, Svetochev V, Haug T (2000) Feeding habits of harp (Phoca groenlandica) and hooded seals (Cystophora cristata) during late winter, spring and early summer in the Greenland Sea. NAMMCO Sci Publ 2: $40-49$

- Radloff FGT, du Toit JT (2004) Large predators and their prey in a southern African savanna: a predator's size determines its prey size range. J Anim Ecol 73:410-423

> Rose GA (2004) Reconciling overfishing and climate change with stock dynamics of Atlantic cod (Gadus morhua) over 500 years. Can J Fish Aquat Sci 61:1553-1557 
Sergeant DE (1991) Harp seals, man, and ice. Can Spec Publ Fish Aquat Sci 114:1-153

Sinclair AF, Murawski SA (1997) Why have groundfish stocks declined? In: Boreman J, Nakashima BS, Wilson JA, Kendall RL (eds) Northwest Atlantic groundfish: perspectives on a fishery collapse. American Fishery Society, Bethesda, MD, p 71-93

Sjare B, Stenson GB, Healy B (2004) Changes in the reproductive parameters of female harp seals (Pagophilus groenlandicus) in the Northwest Atlantic. Canadian Science Advisory Secretariat Research Document 2004/107

Stenson GB, Perry EA (2001) Incorporating uncertainty into estimates of Atlantic cod (Gadus morhua), Capelin (Mallotus villosus), and Arctic cod (Boregogodus saida) consumption by harp seals (Pagophilus groenlandicus) in NAFO Divisions 2J3KL. Canadian Science Advisory Secretariat Research Document 2001/074

Stenson GB, Sjare B (1997) Seasonal distribution of harp seals, Phoca groenlandica, in the northwest Atlantic. ICES CM CC:10. ICES, Copenhagen

Stenson GB, Myers RA, Ni IH, Warren WG (1997) Pup production and population growth of hooded seals

Editorial responsibility: Steve Dawson,

Dunedin, New Zealand
(Cystophora cristata) near Newfoundland, Canada. Can J Fish Aquat Sci 54(Suppl 1):209-216

Stenson GB, Hammill MO, Kingsley MCS, Sjare B, Warren WG, Myers RA (2002) Is there evidence of increased pup production in the northwest Atlantic harp seals, Pagophilus groenlandicus? ICES J Mar Sci 59:81-92

Tucker S (2007) Defining sources of variation in diets of northern phocids using stable isotopes and quantitative fatty acid signature analysis. PhD thesis, Dalhousie University, Halifax

> Tucker S, Bowen WD, Iverson SJ (2007) Dimensions of diet segregation in grey seals Halichoerus grypus revealed through stable isotopes of carbon $\left(\delta^{13} \mathrm{C}\right)$ and nitrogen $\left(\delta^{15} \mathrm{~N}\right)$. Mar Ecol Prog Ser 339:271-282

Tucker S, Bowen WD, Iverson SJ (2008) Convergence of diet estimates derived from fatty acids and stable isotopes within individual grey seals. Mar Ecol Prog Ser 354: $267-276$

Tucker S, Bowen WD, Iverson SJ, Stenson GB (2009) Intrinsic and extrinsic sources of variation in the diets of harp and hooded seals revealed by fatty acid profiles. Can J Zool 87:139-151

Submitted: August 22, 2008; Accepted: March 6, 2009 Proofs received from author(s): May 13, 2009 